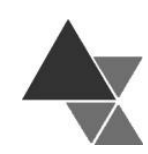

\title{
Influência do Programa Bolsa Família no índice antropométrico peso para idade das crianças beneficiárias em uma cidade no interior de Minas Gerais
} (Brasil)

\author{
Mariana Cristina Silva Santos ${ }^{1}$, Eduardo Bearzoti $^{2}$, Cláudia Aparecida Márliere $^{3}$ e \\ Palmira de Fátima Bonolo 4
}

Objetivo: avaliar a influência do Programa Bolsa Família (PBF) no perfil nutricional de crianças beneficiárias, no município de Ouro Preto, MG, Brasil, por meio do índice antropométrico peso para idade (P/I). Métodos: Estudo epidemiológico analítico, realizado a partir de registros contidos no Sistema de Vigilância Alimentar e Nutricional (SISVAN) e no Cadastro Único do Governo Federal (CadÚnico), referentes a crianças com até 84 meses; e os efeitos das variáveis foram estimados por meio do Modelo Linear de Efeito Misto. Resultados: observou-se P/I elevado de $15,4 \%$ na primeira avaliação e de $8,3 \%$ na segunda; sendo que a redução média foi significativa, de 0,09 no valor do escore $Z$ a cada 100 dias de exposição ao PBF. Conclusão: o tempo de permanência no PBF pode contribuir de maneira significante no estado nutricional das crianças.

Palavras-chave: Programa Bolsa Família; Avaliação em Saúde; Segurança Alimentar e Nutricional.

\section{Influence of the Bolsa Família conditional cash transfer program in anthropometric index weight-for-age for children in Brazilian city}

Objective: Evaluate the influence of the Bolsa Familia Program (BFP) on the nutritional profile of beneficiary children, in the city of Ouro Preto MG, Brazil, through anthropometric index weight for age. Methods: Analytical epidemiological study, conducted from records in the Food and Nutrition Surveillance System (SISVAN) and the Sigle Registry of the Federal Government (CadÚnico), related to children up to 84 months old, and the effects of the variables were estimated by the Linear Mixed-Effect Model. Results: It was possible to observe a high weight for age, $15.4 \%$, in the first evaluation, and $8.3 \%$ in the second; the average reduction was significant, of 0.09 score $\mathrm{z}$ at each 100 days of exposure to the BFP. Conclusion: The results indicate that the residence time in the BFP can significantly contribute to improve the anthropometric index weight for age of beneficiary children.

Keywords: Bolsa Família Program; Health Evaluation; Food and Nutrition Security.

\footnotetext{
${ }^{1}$ Nutricionista. Mestre pelo Programa de Pós-graduação em Saúde e Nutrição - Universidade Federal de Ouro Preto - Brasil. E-mail: santos.marianacs@ggmail.com. ID ORCID: https://orcid.org/0000-0002-4962-8769. Endereço para correspondência: Rua dos Aimorés, 1451 - Lourdes, Belo Horizonte - MG, CEP: 30140-071

${ }^{2}$ Estatístico. Doutor em Agronomia Genética. Departamento de Estatística - Universidade Federal de Ouro Preto - Ouro Preto, MG - Brasil. E-mail: edbearzoti@yahoo.com.br; ID ORCID: https://orcid.org/0000-0002$8313-0577$

${ }^{8}$ Nutricionista. Doutora em Impacto Ambiental. Departamento de Nutrição Clínica e Social da Escola de Nutrição - Universidade Federal de Ouro Preto - Ouro Preto, MG - Brasil. E-mail: calmarliere@ @yahoo.com.br. ID ${ }^{3}$ Nutricionista. Doutora em Impacto Ambiental. D
ORCID: https://orcid.org/0000-0003-1513-9868

${ }^{4}$ Médica. Doutora em Saúde Pública. Departamento de Medicina Preventiva e Social da Faculdade de Medicina - Universidade Federal de Minas Gerais - Belo Horizonte MG - Brasil. E-mail: pfbonolo@gmail.com. ID

ORCID: https://orcid.org/0000-0003-2744-7139
} 


\section{INTRODUÇÃO}

Os programas de transferência de renda de natureza não-contributiva constituem mecanismos diretos de redistribuição de renda que refletem na erradicação da pobreza e na redução substancial dos níveis de desigualdade ${ }^{[1]}$. Existentes desde a década de 1990, esses programas tornaram-se o principal instrumento social na maioria dos países latinoamericanos e Caribe como mecanismo importante para o acesso à educação, saúde e a segurança alimentar e nutricional das famílias ${ }^{[2,3]}$.

No Brasil, o Programa Bolsa Família (PBF) foi criado em 2004 e é uma das principais ações governamentais de desenvolvimento de programas de transferência condicionada de renda, constituindo um pilar importante de combate à pobreza extrema ${ }^{[4]}$. Por meio do Cadastro Único do Governo para Programas Sociais (CadÚnico) é possível realizar a identificação e caracterização das famílias de baixa renda, sendo que o benefício distribuído é calculado observando-se critérios de renda e/ou composição familiar o que permite maior abrangência às famílias que se encontram em situação de vulnerabilidade social[ $[$ ].

As condicionalidades do $\mathrm{PBF}$ reforçam o exercício de direitos básicos e ampliam as possibilidades de acesso aos serviços de saúde e educação, o que contribui para elevar o grau de efetivação dos direitos sociais dos beneficiários ${ }^{[]}$. $\mathrm{O}$ PBF age sobre os determinantes sociais em saúde podendo contribuir para a diminuição da mortalidade infantil, especialmente por mortes atribuíveis a desnutrição e diarreia[7]. Essas alterações e/ou distúrbios podem ser acompanhadas pelos profissionais de saúde da Atenção Primária à Saúde (APS) por meio do calendário de vacinação e da avaliação do crescimento ${ }^{[8]}$.

A avaliação do PBF quanto à situação ligada à saúde de beneficiários têm mostrado bons resultados na segurança alimentar, gastos financeiros com alimentação[9,10] e no estado nutricional[11]. Evidencia-se ainda, que as crianças cadastradas no PBF estão propensas a ter índices antropométricos, especialmente peso para idade $(\mathrm{P} / \mathrm{I})$ e altura para idade $(\mathrm{A} / \mathrm{I})$, mais adequados do que aquelas que não se beneficiam do $\mathrm{PBF}{ }^{[12]}$.

Ademais, no Brasil, a vigilância alimentar e nutricional, promovido pelo Sistema de Vigilância Alimentar e Nutricional (SISVAN), insere-se como importante diretriz da Política Nacional de Alimentação e Nutricional (PNAN) ${ }^{[13]}$. Nesse contexto, as abordagens dessas políticas públicas combinadas podem exercer interferências positivas nas condições de vida da população beneficiária.

Desse modo, o PBF desempenha ações no ambiente mais imediato das famílias, bem como em todos os níveis ambientais e sociais o que pode ocasionar mudanças em curto e/ou longo prazo nos grupos familiares e no estado nutricional[14]. O objetivo deste estudo foi analisar a influência do PBF no índice antropométrico $\mathrm{P} / \mathrm{I}$ de crianças beneficiárias no município de Ouro Preto-MG contribuindo com uma metodologia de avaliação do PBF.

\section{METODOLOGIA}

Trata-se de um estudo epidemiológico, retrospectivo e quantitativo realizado com dados referentes ao acompanhamento da condicionalidade saúde do PBF, no município de Ouro Preto - MG. A condicionalidade inclui as crianças menores de sete anos e o acompanhamento do estado nutricional. A fonte dos dados constituiu-se por informações consolidados provenientes do Sistema de Vigilância Alimentar e Nutricional (SISVAN - Bolsa Família) e do CadÚnico. A população de beneficiários pesquisada foi avaliada durante o período de 2008 a 2013. A determinação da amostra baseou-se no método de amostragem intencional ou não-aleatória por meio da listagem fornecida pela Secretaria Municipal de Saúde de crianças de zero a sete anos 
ativas no PBF até o final do segundo semestre de 2013 ( $n=1353)$. O critério de inclusão considerou a existência do registro de uma avaliação nutricional de entrada e pelo menos uma avaliação de seguimento do estado nutricional.

Para avaliação do perfil antropométrico foram coletadas informações referentes ao peso, comprimento/estatura, idade e sexo. Foram coletadas também informações sociodemográficas referente ao responsável familiar como idade, sexo, cor/raça e escolaridade, e também variáveis referentes ao domicílio (número de pessoas residentes) e ao tempo de exposição (TE) ao PBF. Devido à inconsistência e aos valores biologicamente implausíveis referentes à altura, resultantes do preenchimento, esse dado não foi considerado para efeito de determinação de escores, sendo utilizado apenas o indicador peso por idade $(\mathrm{P} / \mathrm{I})$. As variáveis categóricas foram descritas em frequência absoluta $\mathrm{e}$ relativa e as contínuas em média (desvio-padrão) e mediana (mínimo e máximo).

A determinação do escore $Z$ para o índice antropométrico $\mathrm{P} / \mathrm{I}$ foi realizado por meio do software WHO ANTHRO e WHO ANTHRO PLUS (versão 3.2.2 e 1.0.4) preconizado pelo Ministério da Saúde e Organização Mundial de Saúde (WHO, 2006 e 2007) [15]. Para melhor avaliar a influência do PBF no índice antropométrico $\mathrm{P} / \mathrm{I}$, as variáveis foram ajustadas a um modelo de regressão misto, ou seja, um modelo contendo efeitos fixos e aleatórios, que considera sequência temporal de duas ou mais observações, com estrutura desbalanceada (medida em cada indivíduo observado em tempos diferentes) e aninhada dentro de um mesmo indivíduo [16,17].

A variável dependente (peso para idade) foi avaliada quanto ao efeito relativo à criança como de natureza aleatória e as demais coráveis de natureza fixa. $\mathrm{Na}$ comparação para adequabilidade dos modelos lineares, foi utilizado o teste $F$ com dois graus de liberdade para a variável raça no ajuste do modelo ${ }^{[18]}$. Para a seleção das covariáveis a serem mantidas no modelo misto ajustado, o método de “forward" [19] foi empregado, utilizando um nível de significância de $5 \%$. Os interceptos aleatórios de cada indivíduo foram mantidos no método.

Para o tratamento e tabulação dos dados foram utilizados os softwares Epi Info para a tabulação dos dados e para as análises estatísticas utilizou-se o programa R. A pesquisa empregou dados exclusivamente de origem secundária, sem menção a informações que possam identificar os indivíduos que formaram a base de dados. Além disso, a pesquisa foi aprovada pelo Comitê de Ética e Pesquisa da Universidade Federal de Ouro Preto CEP UFOP sob o parecer no 527.587 e Certificação de Apresentação para Apreciação Ética CAAE no 26861314.9.0000.5150 e, portanto, em plena conformidade com a Resolução do Conselho Nacional de Saúde (CNS) no 466/2012[20].

\section{RESULTADOS E DISCUSSÃO}

De acordo com o critério de inclusão, 29 $(2,14 \%)$ crianças tinham dados com um registro de entrada e pelo menos mais um de seguimento. Dessas, $17(58,6 \%)$ tinham idade de cinco até 60 meses e 12 crianças $(41,4 \%)$ com idade entre cinco e sete anos, sendo que $51,7 \%$ eram do sexo feminino. Quanto ao responsável familiar (RF), majoritariamente eram mulheres sendo que 41,4\% eram de cor negra e mais da metade $(69 \%)$ possuíam apenas o ensino fundamental completo (Tabela 1 ). 
Tabela 1. Perfil demográfico, antropométrico e de participação no Programa Bolsa Família das crianças beneficiárias e características sociodemográficas do responsável familiar, Ouro Preto - MG, 2008-2013.

\begin{tabular}{|c|c|c|}
\hline Características & $\mathbf{n}$ & $\%$ \\
\hline Faixa etária (meses) & 29 & \\
\hline $5-60$ & 17 & 58,6 \\
\hline $61-84$ & 12 & 41,4 \\
\hline Sexo da criança & 29 & \\
\hline Feminino & 15 & 51,7 \\
\hline Masculino & 14 & 48,3 \\
\hline Tempo de exposição ao PBF* & 29 & \\
\hline$\leq 180$ dias & 16 & 55,2 \\
\hline$>180$ dias & 13 & 44,8 \\
\hline P/I* (1 ${ }^{a}$ Avaliação antropométrica) & 27 & \\
\hline Peso adequado & 23 & 84,6 \\
\hline Peso elevado & 4 & 15,4 \\
\hline P/I* (2 Avaliação antropométrica) & 24 & \\
\hline Peso adequado & 22 & 91,7 \\
\hline Peso elevado & 2 & 8,3 \\
\hline Sexo do RF** & 29 & \\
\hline Feminino & 28 & 96,6 \\
\hline Masculino & 1 & 3,4 \\
\hline Cor da pele do $\mathrm{RF}^{* *}$ & 29 & \\
\hline Negra & 12 & 41,4 \\
\hline Parda & 10 & 34,5 \\
\hline Branca/amarela & 7 & 24,1 \\
\hline Escolaridade do RF** & 29 & \\
\hline Ensino fundamental completo & 20 & 69,0 \\
\hline Ensino médio & 9 & 31,0 \\
\hline Idade*** do RF (anos) & 29 & \\
\hline$<35$ & 16 & 55,2 \\
\hline$\geq 35$ & 13 & 44,8 \\
\hline Número de moradores no domicilio & 29 & \\
\hline $1-3$ & 12 & 41,4 \\
\hline$\geq 4$ & 17 & 58,6 \\
\hline
\end{tabular}

*P/I: peso para idade/**RF: Responsável familiar/ ${ }^{* * *}$ Categorizado por meio do valor da mediana

$\mathrm{Na}$ primeira avaliação, $15,4 \%$ das crianças apresentaram P/I elevado e, 8,3\% na avaliação de seguimento, não sendo detectado baixo peso para idade nesta população. 
A Tabela 2 mostra que as medidas de escore $Z$ para a primeira avaliação de entrada (1 $\underline{a}$ avaliação) das crianças apresentaram desvios-padrão acima de dois, indicando P/I elevado. Além disso, possuíam tempo de exposição (TE) médio ao PBF de 161 dias, com mediana igual a zero (amplitude zero a 729).

Para a avaliação de seguimento, ou seja, a segunda avaliação, o TE médio das crianças foi de
628 dias $( \pm 69,9)$, mediana de 559 (amplitude 206 a 1643).

Por conseguinte, as crianças apresentaram assimetria à direita ou positiva para o TE com seis meses e 24 dias a quatro anos e seis meses de participação no PBF. As crianças beneficiárias ainda demonstraram uma média de 4,9 moradores por domicílio (Tabela 2).

Tabela 2. Caracterização do tempo de exposição ao Programa Bolsa Família, distribuição dos valores de escore $Z$ para $\mathrm{P} / \mathrm{I}$, idade do responsável familiar e moradores no domicílio, Ouro Preto - MG, 2008-2013.

\begin{tabular}{|c|c|c|}
\hline Variáveis & Média $\pm D P * * *$ & Mediana (mínimo e máximo) \\
\hline TE* (1a avaliação) & $161 \pm 38,8$ & $0(0-729)$ \\
\hline TE* (2 $\underline{a}$ avaliação) & $628 \pm 69,9$ & $559(206-1643)$ \\
\hline Peso para idade ( $1^{\text {a }}$ avaliação) & $0,50 \pm 0,27$ & $0,15(-2,0-+3,0)$ \\
\hline Peso para idade (2a avaliação) & $0,16 \pm 0,22$ & $0,19(-2,0-+2,0)$ \\
\hline Idade $\mathrm{RF}^{* *}$ & $37,5 \pm 7,9$ & $35,5(27-60)$ \\
\hline Número de moradores & $4,9 \pm 1,3$ & $5(3-9)$ \\
\hline
\end{tabular}

A 1a e 2 avaliações contribuíram com 51 observações para o modelo. A Tabela 3 mostra seu ajuste final e indica que o TE ao PBF contribuiu de forma negativa e significante, o que indica que a cada 100 dias de exposição há uma redução de 0,09 no valor do escore $Z$ do $\mathrm{P} / \mathrm{I}$. As demais covariáveis não mostraram efeito significativo, inclusive para a variável cor da pele que obteve teste $F=1,246$ e $p$ valor $=0,308$.

Tabela 3. Resultados da análise do modelo de efeitos mistos segundo o índice antropométrico peso para idade de crianças beneficiárias

Variação no Índice Peso por Idade (escore $Z$ )

\begin{tabular}{|c|c|c|c|}
\hline & \multicolumn{3}{|c|}{$\checkmark$ ariaçao no Indice Peso por Idade (escore } \\
\hline & Estimativa & Erro Padrão & $p$-valor \\
\hline \multicolumn{4}{|l|}{ Efeito Aleatório } \\
\hline Intercepto & 0,7293 & 0,2663 & 0,012 \\
\hline \multicolumn{4}{|l|}{ Efeito Fixo } \\
\hline Tempo de exposição ao PBF* & $-0,0009$ & 0,0004 & 0,016 \\
\hline Idade do RF** & 0,018 & 0,0271 & 0,498 \\
\hline Escolaridade do RF** & 0,348 & 0,5421 & 0,527 \\
\hline Número de moradores & $-0,073$ & 0,1609 & 0,656 \\
\hline
\end{tabular}


Segundo o Censo Demográfico (2010) e o Relatório de Informações Sociais do Ministério da Economia, a cidade de Ouro Preto apresentou no ano de 2013 2,3\% de pessoas em extrema pobreza e elegíveis a receber o benefício. Em 2020, aproximadamente $3,3 \%$ da população em extrema pobreza do município foram elegíveis. A efetivação no município ocorreu dois anos após a implementação a nível nacional, no ano de 2006, e no ano de 2014 beneficiou 3.022 famílias. Até o primeiro semestre do ano de 2020, o benefício alcançou uma cobertura de $90,3 \%{ }^{[21,22]}$.

Os resultados do estudo confirmam que mais da metade das crianças convivem com o responsável familiar de maioria da cor da pele preta, baixa escolaridade sendo que essa foi uma situação similar encontrada nos estudos realizados Sousa[23] e Saldiva ${ }^{[24]}$ que avaliaram o perfil socioeconômico de familias beneficiárias. A alta concordância com a titularidade feminina do benefício ratifica que o benefício financeiro amplia a autonomia das titulares nas decisões de compra, pois as mulheres são tradicionalmente responsáveis pela distribuição, preparo e cuidados dos alimentos da família [25]. No entanto, estudos indicam que a ampliação das decisões exclusivas das mulheres sobre as questões relativas às crianças e a casa podem interferir nos ganhos de equidade nas relações de gênero e reforça a naturalização da atividade de cuidado como uma tarefa exclusivamente feminina ${ }^{[26]}$.

O percentual de famílias que possuíam menos de um ano de tempo de exposição ao PBF foi ligeiramente superior ao encontrado por Oliveira[27], que apontou o percentual de 48,2\%. Além disso, a média do número de moradores no domicilio $(4,9)$ foi similar ao mesmo estudo $(5,0)$ o que indica que famílias mais numerosas podem ter maior vulnerabilidade social e econômica.

Analisando a interpretação das médias de escores- $Z$, em uma população com distribuição normal, a prevalência esperada de déficit nutricionais, ou seja, escores- $Z$ inferiores a $-2,00$, os desvios padrão são de até $2,28 \%$. No presente estudo, a interpretação das médias de escores- $Z$ foi próxima de 0,00 , tanto para 1a como para a 2a avaliação das crianças. No entanto, ao analisar o índice antropométrico, houve um percentual de peso elevado para idade em $15,4 \%$ das crianças, evidenciado o excesso de peso como um problema eminente em regiões caracterizadas pela transição nutricional e em populações de baixa renda ${ }^{[28,29,30]}$.

$\mathrm{O}$ aumento de peso para crianças tem sido relatado em populações beneficiárias do $\mathrm{PBF}^{[31]}$. Embora não seja possível inferir causas do risco elevado de sobrepeso, as crianças em condições de pobreza e extrema pobreza, características dos beneficiários, estão mais expostas aos alimentos de alta densidade energética e baixo valor nutricional acessíveis, em sua maioria, a preços baixos. Esse fator contribui fortemente para o aumento da insegurança alimentar e nutricional dessas famílias e, consequentemente para as crianças, que consomem menos frutas, verduras e legumes e mais alimentos ultraprocessados, o que contribui para o aumento de peso[32,33].

Apesar disso, ao se comparar as duas avaliações (primeira de entrada e a segunda de seguimento), apesar da média do índice P/I desse estudo estar dentro da faixa considerada adequada, foi possível observar a significativa redução do índice peso para idade nas crianças estudadas. Observou-se que as médias do $\mathrm{P} / \mathrm{I}$ foram significantes para o tempo de exposição, indicando que o PBF pode contribuir na mudança deste índice. Camelo ${ }^{[34]}$ relata melhora na tendência das crianças em saírem de sobrepeso para o peso adequado para idade já que o PBF atua na redução desses índices devido à exigência do acompanhamento nutricional. E embora a pobreza tenha, ainda, efeitos desastrosos sobre a vida dos indivíduos, principalmente entre as crianças da primeira infância, a atenção à saúde, nutricional e educacional permite implementar estratégias mais amplas de articulação para o desenvolvimento econômico e social de uma população[10].

As limitações que podem ser elencadas nesse estudo incluem a não possibilidade de se utilizar um grupo de comparação como, por exemplo, na estimativa frequentemente utilizada do propensity score[34]. Isso se justifica devido ao fato de que a única fonte de dados disponível foi o SISVAN - Bolsa Família e o Cadúnico de famílias sem informações completas do estado nutricional anterior. Além do mais, o uso exclusivo do P/I limita o diagnóstico nutricional, uma vez que este índice reflete a situação 
global, porém não diferencia o comprometimento atual ou agudo dos processos crônicos ${ }^{[35]}$. Assim, para um diagnóstico mais preciso deve-se utilizar outros índices como peso para comprimento/estatura, altura por idade e índice de massa corporal por idade, entretanto outros autores avaliaram o estado nutricional somente com o índice

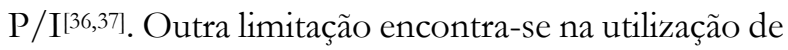
dados secundários em que pode ocorrer descontinuidade das informações e a não precisão de medidas, por muitas vezes serem coletados com a finalidade não específica para a pesquisa e análise de dados e que pode ter contribuído para a não viabilidade de dados referente à altura[ ${ }^{38]}$. Finalmente, o estudo utilizou-se de uma amostra intencional, o que permite a interpretação dos achados somente para a população desse estudo.

No entanto, a possível contribuição desse estudo pauta-se na sinalização positiva da associação do tempo de exposição ao PBF, por meio da transferência condicionada de renda, e a diminuição quantitativa do escore- $Z$ no índice peso para idade. Através de uma metodologia de análise estatística aplicável, o modelo específico mostrou-se adequado para essa amostra refletindo um possível subsídio para embasar discussões em relação à manutenção e ampliação do Programa no município. O fortalecimento da cobertura da população-alvo (elegíveis) ao PBF constitui um ponto importante no combate à fome, erradicação da pobreza, emancipação das famílias ${ }^{[39]}$ e melhora da insegurança alimentar e nutricional das famílias pobres e extremamente pobres.

\section{CONCLUSÃO}

Com o objetivo de analisar a influência do PBF no $\mathrm{P} / \mathrm{I}$ por meio de uma metodologia de modelos lineares, os resultados desse estudo demonstram que a avaliação do PBF por meio do modelo lineares mistos para índices antropométricos pode ser factível para próximos estudos e outros autores o utilizam satisfatoriamente para avaliação do índice antropométrico $\mathrm{P} / \mathrm{I}$ em análises com regressões lineares ${ }^{[32]}$. Apesar do acompanhamento nutricional ser de caráter obrigatório, destaca-se que a ausência das informações não constitui impedimento para que a família seja considerada inclusa no PBF[33]. Reconhece- se como imprescindível a melhora na qualidade dos registros como forma de integração com outras bases de informação para permitir avaliações de impacto das condicionalidades.

Contudo, as condicionalidades do PBF perpassam por atuações intersetoriais dentro do município que requerem articulação e monitoramento contínuo. Faz-se necessário pontuar também que outras ações multisetoriais configuram importantes estratégias na promoção da saúde e qualidade de vida, desde as estratégias que promovem o acesso à água e ao esgotamento sanitário, programas de moradia e infraestrutura até as que promovem as ações de promoção da alimentação saudável que perpassem a regulamentação da propaganda de alimentos e a educação nutricional voltada para população em vulnerabilidade social. A implementação e operacionalização racional de políticas públicas no país precisam ser reforçadas e atuarem de forma intersetorial aos programas de transferência condicionada de renda, de modo a permitir aos beneficiários o acesso a alimentos e modo regular, contínuo e saudável garantindo também o Direito Humano à Alimentação Adequada.

Além disso, outros estudos epidemiológicos que possam analisar a diminuição ou aumento dos escores- $Z$ nos índices antropométricos de crianças beneficiárias são sugeridos como forma também de se investigar as mudanças nos padrões alimentares e no perfil nutricional das crianças beneficiárias, mas principalmente do consumo alimentar da família. Acrescenta-se a esse cenário, a importância de promover o diálogo sobre o contexto de insegurança alimentar e nutricional e a transição nutricional que vive a população brasileira.

\section{REFERÊNCIAS}

[1] Georges R. Retratos da desigualdade no Brasil e na América Latina. In: Siqueira IR, Esteves P (orgs) O desenvolvimento que queremos: Bolsa Família como aprendizado para uma nova agenda. Rio de Janeiro: Editora PUC - Rio; 2018.

[2] Soares FV, Soares S, Medeiros M, Osório RG. Programas de transferência de renda no Brasil: impactos sobre a desigualdade [Internet]. 2006 [acesso em 2021 set 
20]. Textos para discussão (TD) 1228. Brasília: Instituto de Pesquisa Econômica Aplicada (IPEA); 2006. Disponível em: http:/ /www.ipea.gov.br

[3] Leroy JL, Garcia-Guerra A, Garcia R, Dominguez C, Rivera J, Neufeld LM. The Opportunity program increase the linear growth of children enrolled at young ages in urban Mexico. J. Nutr. 2008;138:793-798.

[4] Brasil. Lei Ordinária no 10.836, de 9 de janeiro de 2004. Cria o Programa Bolsa Família, altera a Lei no 10.689 e dá outras providências. Diário Oficial da República Federativa do Brasil. Brasília; 2004.

[5] Brasil. Ministério do Desenvolvimento Social e Combate à Fome. Programas de transferência direta de renda com condicionalidades e Cadastro Único para Programas Sociais do Governo: composição de valores. 2020 [acesso em 2020 abr 7]. Disponível em: http://www.mds.gov.br/bolsafamilia/beneficios/compo sicao-de-valores

[6] Brasil. Ministério da Saúde. Secretaria de Atenção à Saúde. Departamento de Atenção Básica. Alimentação e nutrição para famílias do Programa Bolsa Família: manual para os agentes comunitários de saúde. 2007 [acesso em 2020 abr 26]. Disponível em: http://189.28.128.100/nutricao/docs/geral/manual_acs _2010_2011.pdf

[7] Rasella D, Aquino R, Santos CAT, Paes-Sousa R, Barreto ML. Effect of a conditional cash transfer programme on childhood mortality: a nationwide analysis of Brazilian municipalities. Lancet. 2013;382(9886):57-64.

[8] Saldanha LF, Lagares ÉB, Fonseca PC, Anastácio LR. Estado nutricional de crianças beneficiárias do Programa Bolsa Família acompanhadas pelo Sistema de Vigilância Alimentar e Nutricional no Estado de Minas Gerais. Rev. Médica Minas Gerais [Internet]. 2014 [acesso em 2020 abr 26]. 2014;24(4):478-485. Disponível em: http://www.gnresearch.org/doi/10.5935/22383182.20140139

[9] Sousa CC, Goncalves LA, Lopes Mikael J, Toledo, VRP. Segurança alimentar, renda e Programa Bolsa Família: estudo de coorte em municípios do interior da Paraíba, Brasil, 2005-2011. Cad. Saúde Pública [Internet]. 2014 [acesso em 2015 jan 16]. 2014;30(2):393-402.

[10] Suzart AS, Ferreira AP, Suzart AS, Ferreira AP. Avaliação do Programa Bolsa Família (PBF) na segurança alimentar e nutricional das famílias beneficiadas, Vitória da Conquista, BA. Interações [Internet]. 2018 [acesso em 2020 abr 15]. 2018;19(3):585-595.
[11] Brasil. Ministério do Desenvolvimento Social e Combate à Fome (MDS). Chamada Nutricional: um estudo sobre a situação nutricional das crianças do semiárido brasileiro. Brasília: MDS; 2006.

[12] Paes-Sousa R, Santos LMP, Miasaki ES. Effects of conditional cash transfer programme on child nutrition in Brazil. Bull Word Health Organ. 2011;89(7):496-503.

[13] Brasil. Ministério da Saúde. Secretaria de Atenção à Saúde. Departamento de Atenção Básica. Política Nacional de Alimentação e Nutrição. Brasília: MS; 2013.

[14] Freire VRBP, Silva SSC, Cavalcante LIC, Pontes FAR. Programa Bolsa Família como estratégia de combate à pobreza em dissertações e teses no Brasil. Revista Estudos e Pesquisas em Psicologia. 2013,13(13):990-1009.

[15] Brasil. Ministério da Saúde. Orientações para Coleta e Análise de Dados Antropométricos em Serviços de Saúde. Norma Técnica do Sistema de Vigilância Alimentar e Nutricional - SISVAN. Brasília: MS; 2011.

[16] McCulloch CE, Searle SR. Generalized, Linear, and Mixed Models. Led. New York: Wiley; 2001.

[17] Fausto MA, Carneiro M, Antunes CMF, Pinto JA, Colosimo EA. O modelo de regressão linear misto para dados longitudinais: uma aplicação na análise de dados antropométricos desbalanceados. Cad. Saúde Pública. 2008;24(3):513-524.

[18] Gaudio AC, Zandonade E. Proposição, validação e análise dos modelos que correlacionam estrutura química e atividade biológica. Química Nova. 2001;24(5):658-571.

[19] Draper N, Smith H. Applied Regression Analysis. 3. ed. New York: John Wiley \& Sons; 1998.

[20] Brasil. Ministério da Saúde. Resolução no 466, de 12 de dezembro de 2012. Aprova diretrizes e normas regulamentadoras de pesquisas envolvendo seres humanos. Brasília; 2012.

[21] Instituto Brasileiro de Geografia e Estatística - IBGE Cidades. Minas Gerais. Ouro Preto. 2019 [acesso em 2020 fev 20]. Disponível em: http://cidades.ibge.gov.br/xtras/home.php

[22] Brasil. Ministério do Desenvolvimento Social e Combate à Fome. Secretária de Avaliação e Gestão da Informação. Relatório de Informações Sociais do Programa Bolsa Família e do Cadastro Único. 2020 [acesso em 2020 fev 20]. Disponível em: 
https://aplicacoes.mds.gov.br/sagi/RIv3/geral/index.ph p? relatorio $=153 \&$ file $=$ entrada

[23] Souza NN, Dias MM, Sperandio N, Franceschini SCC, Priore SE. Perfil socioeconômico e insegurança alimentar e nutricional de famílias beneficiárias do Programa Bolsa Família no município de Viçosa, Estado de Minas Gerais, Brasil, em 2011: um estudo epidemiológico transversal. Epidemiol. Serv. Saúde, 2012;21(4):655-662.

[24] Saldiva SRDM, Silva LFF, Saldiva PHN. Avaliação antropométrica e consumo alimentar em crianças menores de cinco anos residentes em um município da região do semiárido nordestino com cobertura parcial do programa bolsa família. Rev. Nutr. 2010;23(2):221-229.

[25] Cruz LR. O Programa Bolsa Família e perspectivas de gênero: análises transversais. 2016. Anais do XIX Encontro Nacional de Estudos Populacionais. 2016.

[26] Bartholo L, Passos L, Fontoura N. The Family Grant Program, Female Autonomy and Gender Equity: What do national studies indicate? Cadernos Pagu. 2019;(55):e195525.

[27] Oliveira FCC, Cotta RMM, Ribeiro AQ, Sant'ana LFR, Priore SE, Franceschini SCC. Estado nutricional e fatores determinantes do déficit estatural em crianças cadastradas no Programa Bolsa Família. Epidemiol. Serv. Saúde. 2011;20(1):7-18.

[28] Pelegrini A, Silva DAS, Petroski EL, Gaya ACA. Overweight and obesity in seven to nine-year-old Brazilian students: data from the Brazilian Sports Project. Rev. Paul. Pediatr. 2010;28(3):290-295.

[29] Silva DAS. Sobrepeso e obesidade em crianças de cinco a dez anos de idade beneficiárias do Programa Bolsa Família no estado de Sergipe, Brasil. Rev. Paul. Pediatr. 2011;29(4):529-535.

[30] Lima DGD, Freitas AG, Cavalcante JB, Silva IS, Meneguetti DUDO, Silva RPM. Comparação do estado nutricional de crianças beneficiárias do Programa Bolsa Família em diferentes regiões de saúde do Acre. Journal of Amazon Health Science. 2016;2(3).

[31] Lignani JB, Burlandy L, Sichieri R, Salles R. Changes in food consumption among the Programa Bolsa Família participant families in Brazil. Public Health Nutr. 2011;14:785-792.
[32] Giesta JM, Zoche E, Corrêa R da S, Bosa VL. Fatores associados à introdução precoce de alimentos ultraprocessados na alimentação de crianças menores de dois anos. Ciênc. Saúde Coletiva. 2019;24:2387-2397.

[33] Louzada MLC, Martins APB, Canella DS, Baraldi LG, Levy RB, Claro RM et al. Ultra-processed foods and the nutritional dietary profile in Brazil. Rev. Saúde Pública. 2015;49(38):1-11.

[34] Camelo RS, Tavares PA, Saiani CCS. Alimentação, nutrição e saúde em programas de transferência de renda: evidências do Programa Bolsa Família. Revista Economia. 2009,10(4):685-713.

[35] Brasil. Ministério da Saúde. Departamento de Informática do Sistema Único de Saúde (DATASUS). Notas Técnicas: SISVAN Municipal - estado nutricional dos usuários da atenção básica [Internet]. 2020 [acesso em 2020 fev 10]. Disponível em: http://tabnet.datasus.gov.br/cgi-

win/SISVAN/CNV/notas_sisvan.html

[36] Silva OLO, Lindemann IL, Prado SG, Freitas KC, Souza AS. Vigilância alimentar e nutricional de crianças indígenas menores de cinco anos em Mato Grosso do Sul, 2002-2011. Epidemiol. Serv. Saúde. 2014;23(3):541-546.

[37] Augusto RA, Souza JMP. Efetividade de programa de suplementação alimentar no ganho ponderal de crianças. Rev. Saúde Pública. 2010;44(5):793-801.

[38] Drumond E, Machado CJ, Vasconcelos MR, França E. Utilização de dados secundários do SIM, Sinasc e SIH na produção científica brasileira de 1990 a 2006. Revista Brasileira de Estudos de População. 2009;26(1):7-19.

[39] Kopruszynski CP, Costa VMHM. Programas de transferência condicionada de renda e segurança alimentar e nutricional. Segur. Aliment. Nutr. 2016;23:996-1007. 